TRANSACTIONS OF THE

AMERICAN MATHEMATICAL SOCIETY

Volume 353, Number 2, Pages 733-747

S 0002-9947(00)02735- 5

Article electronically published on October 13, 2000

\title{
BLOCK DIAGONAL POLYNOMIALS
}

\author{
VERÓNICA DIMANT AND RAQUEL GONZALO
}

\begin{abstract}
In this paper we introduce and study a certain class of polynomials in spaces with unconditional finite dimensional decomposition. Some applications to the existence of copies of $\ell_{\infty}$ in spaces of polynomials and to the stabilization of polynomial algebras are given.
\end{abstract}

\section{INTRODUCTION}

Motivated by several problems related to the structure of spaces of polynomials, we introduce and study the class of block diagonal polynomials in the context of spaces with unconditional finite dimensional decomposition. This kind of polynomials will be useful to attack two different problems. On one hand, for many examples in the literature it is noted that the space of homogeneous polynomials contains an isomorphic copy of $\ell_{\infty}$ (conditions to ensure this fact can be found in [12, 13, 9, 14, 10, 16]). Also, it is proved in [9] that there exists a dichotomy between the fact that all polynomials are weakly sequentially continuous and the existence of isomorphic copies of $\ell_{\infty}$ in the space of polynomials. Here we are concerned with the "position" of such isomorphic copies of $\ell_{\infty}$ and the relationship between weak sequential continuity of polynomials and block diagonal polynomials. The other problem is to study the stabilization of polynomial algebras [23]. In this direction, we describe the behavior of the chain of algebras generated by polynomials up to a certain degree in the particular case of Banach spaces with unconditional finite dimensional decomposition.

In Section 1, we introduce the class of block diagonal polynomials and establish some properties of these polynomials. We provide a technique to construct, from a fixed polynomial, a block diagonal polynomial which preserves some properties of the original one. Using this, we prove the equivalence between weak sequential continuity of polynomials and the same property for block diagonal polynomials. Moreover, if there exist polynomials which are not weakly sequentially continuous, this technique turns out to be useful to show how to embed an isomorphic copy of $\ell_{\infty}$ in a certain space of block diagonal polynomials.

The second section is totally devoted to studying the simplest class of block diagonal polynomials in a space with unconditional basis, namely, diagonal polynomials. In the case of real Banach spaces with 1-unconditional basis, considered

Received by the editors July 24, 1998 and, in revised form, July 22, 1999.

2000 Mathematics Subject Classification. Primary 46G20; Secondary 46Bxx.

Key words and phrases. Polynomials, weak sequential continuity, containment of $\ell_{\infty}$.

The first author was partially supported by Instituto de Cooperación Iberoamericano, and the second author was partially supported by PGCYT PB-96-0607.

(C)2000 American Mathematical Society 
as Banach lattices, diagonal polynomials coincide with orthogonally additive polynomials, which were introduced and studied by Sundaresan [24]. We see how weak sequential continuity of diagonal polynomials has a great impact on the behavior of all polynomials when applied to the basis; indeed, the basis turns out to be polynomially null. On the other hand, we give an example which shows that the reciprocal result is not true. Also, we generalize some results given in 24 for $\ell_{p}$ spaces, to the case of spaces in which the basis has a lower estimate.

Finally, in Section 3 we apply some of the results obtained in Section 1 to give the description of the chain of algebras of polynomials generated by polynomials up to a certain degree. We prove that for real Banach spaces not containing $\ell_{1}$, with unconditional finite dimensional decompositions, if there exists a polynomial which is not weakly sequentially continuous then this chain does not stabilize. Moreover, the lowest degree of a polynomial which is not weakly sequentially continuous is the breaking point of the chain. After this point, each element is different from the following one. A similar result was obtained by Hájek [19], under the assumption of the existence of a non-compact operator from the space into some $\ell_{p}$ space. To our knowledge, this assumption is less general than the existence of a polynomial which is not weakly sequentially continuous. In the last part of the article we discuss this problem, relating it to another open problem.

In the sequel we introduce some notations and definitions. Unless stated otherwise, $E$ is a real or a complex Banach space. The space of $k$-homogeneous (continuous) polynomials on $E$ is denoted by $\mathcal{P}\left({ }^{k} E\right)$. The subspace of weak sequential continuous polynomials (respectively, weak sequential continuous at 0 ) is denoted by $\mathcal{P}_{w s c}\left({ }^{k} E\right)$ (resp. $\left.\mathcal{P}_{w s c 0}\left({ }^{k} E\right)\right)$. In general, for any space of polynomials the subindex $w s c$ (resp. wsc0) means the subspace of weak sequential continuous (resp. at 0 ) elements.

We recall some definitions. For $1 \leq p<\infty$, a sequence $\left\{e_{n}\right\}_{n \in \mathbb{N}}$ is said to have a lower $p$-estimate (resp. upper $p$-estimate) if there exists a constant $C>0$ such that

$$
\sum_{n=1}^{\infty}\left|x_{n}\right|^{p} \leq C\left\|\sum_{n=1}^{\infty} x_{n} e_{n}\right\|^{p}
$$

or, respectively,

$$
\sum_{n=1}^{\infty}\left|x_{n}\right|^{p} \geq C\left\|\sum_{n=1}^{\infty} x_{n} e_{n}\right\|^{p} .
$$

A sequence $\left\{e_{n}\right\}_{n \in \mathbb{N}}$ in $E$ is said to be $\mathcal{P}_{k}$-null if $P\left(e_{n}\right) \underset{n \rightarrow \infty}{\longrightarrow} 0$ for all $r$ homogeneous polynomials $P$ with $1 \leq r \leq k$.

\section{BLOCK DIAGONAL POLYNOMIALS}

Throughout this section, $E$ is a Banach space with unconditional finite dimensional decomposition $\left\{E_{n}\right\}_{n \in \mathbb{N}}$ (see 22 for the definition). We denote by $\left\{\Pi_{n}\right\}_{n \in \mathbb{N}}$ the sequence of projections associated to the decomposition; that is,

$$
\Pi_{n}(x)=\sum_{j=1}^{n} x_{j} \quad \forall x=\sum_{j=1}^{\infty} x_{j} \in E, x_{j} \in E_{j} .
$$

Let $J=\left\{n_{j}\right\}_{j \in \mathbb{N}}$ be an ordered infinite set of positive integers. We say that a sequence $\left\{u_{j}\right\}_{j \in \mathbb{N}} \subset E$ is a block sequence with respect to $J$ if $u_{j} \in \operatorname{Im}\left(\Pi_{n_{j}}-\Pi_{n_{j-1}}\right)$, 
for all $j \in \mathbb{N}$. The following lemma generalizes a classical result of spaces with Schauder bases [22, Prop. 1.a.12] to spaces with finite dimensional decomposition (not necessarily unconditional).

Lemma 1.1. Let $E$ be a Banach space with finite dimensional decomposition $\left\{E_{n}\right\}_{n \in \mathbb{N}}$ and let $\left\{x_{k}\right\}_{k \in \mathbb{N}}$ be a seminormalized weakly null sequence on $E$. Then there exist a subsequence $\left\{x_{k_{j}}\right\}_{j \in \mathbb{N}}$ of $\left\{x_{k}\right\}_{k \in \mathbb{N}}$ and a block sequence $\left\{u_{j}\right\}_{j \in \mathbb{N}} \subset E$ such that $\left\|x_{k_{j}}-u_{j}\right\| \underset{j \rightarrow \infty}{\longrightarrow} 0$. Moreover, if $J=\left\{n_{j}\right\}_{j \in \mathbb{N}} \subset \mathbb{N}$ is fixed, we can choose a subsequence $J_{1}$ of $J$ such that $\left\{u_{j}\right\}_{j \in \mathbb{N}}$ is a block sequence with respect to $J_{1}$.

Proof. Our procedure is based on the following two facts:

- If $l \in \mathbb{N}$ is fixed, then $\left\|\Pi_{l}\left(x_{k}\right)\right\| \underset{k \rightarrow \infty}{\longrightarrow} 0$ (since it is a weakly null sequence in a finite dimensional subspace).

- If $k \in \mathbb{N}$ is fixed, then $\left\|x_{k}-\Pi_{l}\left(x_{k}\right)\right\| \underset{l \rightarrow \infty}{\longrightarrow} 0$ (since $\Pi_{l}$ are the projections associated to the decomposition).

Let $x_{k_{1}}=x_{1}$ and $l_{1} \in \mathbb{N}$ be such that $\left\|x_{k_{1}}-\Pi_{l_{1}}\left(x_{k_{1}}\right)\right\|<1$. Let $k_{2}>k_{1}$ be such that $\left\|\Pi_{l_{1}}\left(x_{k_{2}}\right)\right\|<\frac{1}{4}$ and let $l_{2}>l_{1}$ be such that $\left\|x_{k_{2}}-\Pi_{l_{2}}\left(x_{k_{2}}\right)\right\|<\frac{1}{4}$. Thus, $\left\|x_{k_{2}}-\left(\Pi_{l_{2}}-\Pi_{l_{1}}\right)\left(x_{k_{2}}\right)\right\|<\frac{1}{2}$. Consider now $k_{3}>k_{2}$ such that $\left\|\Pi_{l_{2}}\left(x_{k_{3}}\right)\right\|<\frac{1}{6}$ and let $l_{3}>l_{2}$ be such that $\left\|x_{k_{3}}-\Pi_{l_{3}}\left(x_{k_{3}}\right)\right\|<\frac{1}{6}$; thus, $\left\|x_{k_{3}}-\left(\Pi_{l_{3}}-\Pi_{l_{2}}\right)\left(x_{k_{3}}\right)\right\|<\frac{1}{3}$.

Inductively, we obtain a subsequence $\left\{x_{k_{j}}\right\}_{j \in \mathbb{N}}$ of $\left\{x_{k}\right\}_{k \in \mathbb{N}}$ and a block sequence $\left\{u_{j}=\left(\Pi_{l_{j}}-\Pi_{l_{j-1}}\right)\left(x_{k_{j}}\right)\right\}_{j \in \mathbb{N}}$ such that $\left\|x_{k_{j}}-u_{j}\right\| \underset{j \rightarrow \infty}{\longrightarrow} 0$.

Note that if a certain subsequence $J$ of $\mathbb{N}$ is given, in the same way we can choose the indexes $l_{j}$ in $J$; so, the last assertion is proved.

Definition 1.2. Let $J=\left\{n_{j}\right\}_{j \in \mathbb{N}}$ be an increasing sequence of positive integers. For each $j \in \mathbb{N}$, let $\sigma_{j}=\Pi_{n_{j}}-\Pi_{n_{j-1}}$. We define the class of block diagonal $k$-homogeneous polynomials with respect to $J$ as the set

$$
\mathcal{D}_{J}\left({ }^{k} E\right)=\left\{P \in \mathcal{P}\left({ }^{k} E\right): P(x)=\sum_{j=1}^{\infty} P\left(\sigma_{j}(x)\right), \forall x \in E\right\} .
$$

Note that $\sigma_{j}$ depends on the subsequence of integers $J$.

The following proposition will be the key to proving most of the results, since it provides a way to construct, from a certain polynomial, a block diagonal polynomial which preserves some properties of the original one. It is essentially a reformulation of part of the proof of Theorem 13 in [9].

Proposition 1.3. Let $E$ be a Banach space with unconditional finite dimensional decomposition $\left\{E_{n}\right\}_{n \in \mathbb{N}}$ and $P \in \mathcal{P}\left({ }^{k} E\right)$. Then there exists a constant $C>0$ such that for every subsequence $J$ of $\mathbb{N}$,

$$
\sum_{j=1}^{\infty}\left|P\left(\sigma_{j}(x)\right)\right| \leq C\|x\|^{k} \quad \forall x \in E .
$$

Proof. Consider first that $E$ is a complex Banach space. Let $\left\{r_{j}\right\}_{j \in \mathbb{N}}$ be the generalized Rademacher functions introduced by Aron and Globevnik [2]. For each 
$x \in E$ and $j \in \mathbb{N}$, let $\gamma_{j} \in \mathbb{C}$ be such that $P\left(\gamma_{j} \sigma_{j}(x)\right)=\left|P\left(\sigma_{j}(x)\right)\right|$. So we have

$$
\begin{aligned}
\sum_{j=1}^{\infty}\left|P\left(\sigma_{j}(x)\right)\right| & =\sum_{j=1}^{\infty} P\left(\gamma_{j} \sigma_{j}(x)\right)=\int_{0}^{1} P\left(\sum_{j=1}^{\infty} r_{j}(t) \gamma_{j} \sigma_{j}(x)\right) d t \\
& \leq\|P\| \sup _{t \in[0,1]}\left\|\sum_{j=1}^{\infty} r_{j}(t) \gamma_{j} \sigma_{j}(x)\right\|^{k} \leq(2 K)^{k}\|P\|\|x\|^{k}
\end{aligned}
$$

where $K$ is the unconditional constant of the decomposition.

In the case that $E$ is a real Banach space, we consider the complexification $\bar{E}$ of $E$, as in $[5] ;\left\{\bar{E}_{n}\right\}_{n \in \mathbb{N}}$ is an unconditional finite dimensional decomposition of $\bar{E}$. Let $P \in \mathcal{P}\left({ }^{k} E\right)$ be fixed; then there exists a unique extension $\bar{P} \in \mathcal{P}\left({ }^{k} \bar{E}\right)$ with $\|P\| \leq\|\bar{P}\| \leq \frac{(2 k)^{k}}{k !}\|P\|$. Thus,

$$
\sum_{j=1}^{\infty}\left|P\left(\sigma_{j}(x)\right)\right|=\sum_{j=1}^{\infty}\left|\bar{P}\left(\bar{\sigma}_{j}(x)\right)\right| \leq(2 K)^{k}\|\bar{P}\|\|x\|^{k} \leq \frac{(4 k K)^{k}}{k !}\|P\|\|x\|^{k}
$$

where $K$ is the unconditional constant of the decomposition of $\bar{E}$.

Corollary 1.4. Let $E$ be a Banach space with unconditional finite dimensional decomposition and $J$ a subsequence of $\mathbb{N}$. Then $\mathcal{D}_{J}\left({ }^{k} E\right)$ is a complemented subspace of $\mathcal{P}\left({ }^{k} E\right)$.

Proof. It is clear that $\mathcal{D}_{J}\left({ }^{k} E\right)$ is a linear subspace of $\mathcal{P}\left({ }^{k} E\right)$. Let $\Pi: \mathcal{P}\left({ }^{k} E\right) \rightarrow$ $\mathcal{D}_{J}\left({ }^{k} E\right)$ be defined by $\Pi(P)(x)=\sum_{j=1}^{\infty} P\left(\sigma_{j}(x)\right)$. By Proposition 1.3, $\Pi$ is a well defined and continuous operator. Obviously, $\Pi(P)=P$ for every $P \in \mathcal{D}_{J}\left({ }^{k} E\right)$; so, $\Pi$ is a projection whose image is $\mathcal{D}_{J}\left({ }^{k} E\right)$.

In the following proposition we prove that weak sequential continuity of block diagonal polynomials can be replaced by a weaker condition, namely, weak sequential continuity at the origin.

Proposition 1.5. Let $E$ be a Banach space with unconditional finite dimensional decomposition. The following assertions are equivalent:

(i) $\mathcal{D}_{J}\left({ }^{k} E\right)=\mathcal{D}_{J, w s c}\left({ }^{k} E\right)$, for all subsequence $J$ of $\mathbb{N}$.

(ii) $\mathcal{D}_{J}\left({ }^{k} E\right)=\mathcal{D}_{J, w s c 0}\left({ }^{k} E\right)$, for all subsequence $J$ of $\mathbb{N}$.

(iii) $\mathcal{D}_{J}\left({ }^{r} E\right)=\mathcal{D}_{J, w s c 0}\left({ }^{r} E\right)$, for all $1 \leq r \leq k$ and all subsequence $J$ of $\mathbb{N}$.

Proof. Obviously, (i) implies (ii). To see that (ii) implies (iii), assume that there exist a subsequence $J$ of $\mathbb{N}$ and a polynomial $P \in \mathcal{D}_{J}\left({ }^{r} E\right)$ which is not weakly sequentially continuous at $0(1 \leq r<k)$. By Lemma 1.1, there exist $\varepsilon>0$, a subsequence $J_{1}$ of $J$ and a weakly null seminormalized block sequence with respect to $J_{1},\left\{u_{i}\right\}_{i \in \mathbb{N}}$, such that $\left|P\left(u_{i}\right)\right|>\varepsilon$, for all $i \in \mathbb{N}$. Note that $\left\{u_{i}\right\}_{i \in \mathbb{N}}$ is an unconditional basic sequence in $E$.

If $P \in \mathcal{D}_{J}\left({ }^{r} E\right)$ then $P \in \mathcal{D}_{J_{1}}\left({ }^{r} E\right)$. Let $\left\{\sigma_{i}\right\}_{i \in \mathbb{N}}$ the projections associated to $J_{1}$, so

$$
P(x)=\sum_{i=1}^{\infty} P\left(\sigma_{i}(x)\right)
$$


Consider now the sequence $\left\{u_{i}^{*}\right\}_{i \in \mathbb{N}}$ of biorthogonal functionals associated to $\left\{u_{i}\right\}_{i \in \mathbb{N}}$, and define $Q \in \mathcal{P}\left({ }^{k} E\right)$ by

$$
Q(x)=\sum_{i=1}^{\infty} P\left(\sigma_{i}(x)\right)\left(u_{i}^{*}\left(\sigma_{i}(x)\right)\right)^{k-r} .
$$

The polynomial $Q \in \mathcal{D}_{J_{1}}\left({ }^{k} E\right)$ satisfies $Q\left(u_{j}\right)=P\left(u_{j}\right)$, for all $j \in \mathbb{N}$, so $Q$ is not weakly sequentially continuous at 0 , which contradicts (ii).

For the proof that (iii) implies (i), let $P \in \mathcal{D}_{J}\left({ }^{k} E\right)$ and $x_{m} \stackrel{w}{\longrightarrow} x$ in $E$. Then,

$$
P\left(x_{m}\right)=\sum_{j=1}^{k}\left(\begin{array}{l}
k \\
j
\end{array}\right) \widehat{d^{j} P}(x)\left(x_{m}-x\right)+P(x),
$$

where $\widehat{d^{j} P}(x)$ is the $j$-th derivative of $P$ at the point $x$. By using the polarization formula, we obtain that for all $1 \leq l \leq k$,

$$
\stackrel{\vee}{P}\left(x, \ldots, x, \sigma_{j_{1}}(y), \ldots, \sigma_{j_{l}}(y)\right)=0 \quad \text { if the } j_{i} \text { 's are not all equal, }
$$

where $\left\{\sigma_{j}\right\}_{j \in \mathbb{N}}$ are the projections associated to $J$ and $\stackrel{\vee}{P}$ is the $k$-linear symmetric functional associated to $P$. Thus,

$$
\begin{aligned}
\widehat{d^{j} P}(x)(y) & =\widehat{d^{j} P}(x)\left(\sum_{i=1}^{\infty} \sigma_{i}(y)\right)=\sum_{i=1}^{\infty} \stackrel{\vee}{P}\left(x, \ldots, x, \sigma_{i}(y), \ldots, \sigma_{i}(y)\right) \\
& =\sum_{i=1}^{\infty} \widehat{d^{j} P}(x)\left(\sigma_{i}(y)\right),
\end{aligned}
$$

which implies that, for all $x \in E$, the $j$-homogeneous polynomial $\widehat{d^{j} P}(x)$ belongs to $\mathcal{D}_{J}\left({ }^{j} E\right)$, for any $1 \leq j \leq k$. By (iii), $\widehat{d^{j} P}(x)$ is weakly continuous at 0 and $x_{m}-x$ is a weakly null sequence; so, by (1) $P\left(x_{m}\right) \rightarrow P(x)$.

The existence of a polynomial which is not weakly sequentially continuous implies the existence of a block diagonal polynomial which shares the same property, as it is proved in the following proposition.

Proposition 1.6. Let $E$ be a Banach space with unconditional finite dimensional decomposition. If there exists a $k$-homogeneous polynomial which is not weakly sequentially continuous at 0 , then there exist a subsequence $J$ of $\mathbb{N}$ and a block diagonal polynomial with respect to $J$ which is not weakly sequentially continuous at 0 .

Proof. Assume that there exists a $k$-homogeneous polynomial which is not weakly sequentially continuous at 0 . By Lemma 1.1, there exist $\varepsilon>0$, a subsequence $J$ of $\mathbb{N}$ and a weakly null seminormalized block sequence with respect to $J,\left\{u_{j}\right\}_{j \in \mathbb{N}}$, such that $\left|P\left(u_{j}\right)\right|>\varepsilon$, for all $j \in \mathbb{N}$. By Proposition 1.3 .

$$
\tilde{P}(x)=\sum_{j=1}^{\infty} P\left(\sigma_{j}(x)\right)
$$

is a block diagonal polynomial with respect to $J$. Moreover, since $\tilde{P}\left(u_{j}\right)=P\left(u_{j}\right)$, for all $j \in \mathbb{N}$, the polynomial $\tilde{P}$ is not weakly sequentially continuous at 0 . 
As a consequence of Propositions 1.5 and 1.6. we obtain the following corollary. Similar results were established in a non-published addendum to [15] and in [7] 6].

Corollary 1.7. Let $E$ be a Banach space with unconditional finite dimensional decomposition. The following assertions are equivalent:

(i) $\mathcal{P}\left({ }^{k} E\right)=\mathcal{P}_{w s c}\left({ }^{k} E\right)$.

(ii) $\mathcal{P}\left({ }^{k} E\right)=\mathcal{P}_{w s c 0}\left({ }^{k} E\right)$.

(iii) $\mathcal{P}\left({ }^{r} E\right)=\mathcal{P}_{\text {wsc } 0}\left({ }^{r} E\right)$, for all $1 \leq r \leq k$.

The following result has a double interest. On one hand, it shows, assuming the existence of a non-weak sequential continuous polynomial, how to embed $\ell_{\infty}$ in certain spaces of block diagonal polynomials. On the other hand, this result is the key to proving the main result in the last section, since it provides, from the existence of a polynomial which is not weakly sequentially continuous, a polynomial whose restriction to a certain subspace with a basis has a diagonal representation.

Proposition 1.8. Let $E$ be a Banach space with unconditional finite dimensional decomposition. If there exists a $k$-homogeneous polynomial which is not weakly sequentially continuous, then there exist a subsequence $J$ of $\mathbb{N}$, a seminormalized weakly null block sequence with respect to $J,\left\{u_{j}\right\}_{j \in \mathbb{N}}$, and a polynomial $P \in$ $\mathcal{D}_{J}\left({ }^{k} E\right)$, such that

$$
P\left(\sum_{j=1}^{\infty} a_{j} u_{j}\right)=\sum_{j=1}^{\infty} a_{j}^{k} \quad \text { whenever } \sum_{j=1}^{\infty} a_{j} u_{j} \in \overline{\left[\left\{u_{j}\right\}_{j \in \mathbb{N}}\right]} .
$$

Moreover, $\mathcal{D}_{J}\left({ }^{k} E\right)$ contains an isomorphic copy of $\ell_{\infty}$.

Proof. By the previous corollary, there exists a $k$-homogeneous polynomial which is not weakly sequentially continuous at 0 . Consequently, by Proposition 1.6, there exist a subsequence $J$ of $\mathbb{N}$, a seminormalized weakly null block sequence with respect to $J,\left\{u_{j}\right\}_{j \in \mathbb{N}}$, and a polynomial $Q \in \mathcal{D}_{J}\left({ }^{k} E\right)$ such that $\left|Q\left(u_{j}\right)\right|>\varepsilon$, for certain $\varepsilon>0$. We can assume, without loss of generality, that the sequence $\left\{u_{j}\right\}_{j \in \mathbb{N}}$ belongs to the unit ball of $E$. Let $P \in \mathcal{D}_{J}\left({ }^{k} E\right)$ be

$$
P(x)=\sum_{j=1}^{\infty} Q\left(\sigma_{j}(x)\right) Q\left(u_{j}\right)^{-1},
$$

which is well defined by Proposition 1.3 and satisfies (2).

Define $T: \ell_{\infty} \rightarrow \mathcal{D}_{J}\left({ }^{k} E\right)$ by $T(b)(x)=\sum_{j=1}^{\infty} b_{j} P\left(\sigma_{j}(x)\right)$; it is easy to prove that

$$
\|b\|_{\infty} \leq\|T(b)\| \leq C\|b\|_{\infty}
$$

where $C$ is the constant which appears in Proposition 1.3 .

Proposition 1.9. Let $E$ be a Banach space with unconditional finite dimensional decomposition. Let $J$ be subsequence of $\mathbb{N}$ such that there exists a seminormalized block sequence with respect to $J,\left\{u_{j}\right\}_{j \in \mathbb{N}}$, which is not $\mathcal{P}_{k}$-null. Then, the space $\mathcal{D}_{J}\left({ }^{k} E\right)$ contains an isomorphic copy of $\ell_{\infty}$.

Proof. If $\left\{u_{j}\right\}_{j \in \mathbb{N}}$ is not $\mathcal{P}_{k}$-null, then there exist $\varepsilon>0$, a polynomial $P \in \mathcal{P}\left({ }^{r} E\right)$, for some $1 \leq r \leq k$, and a subsequence $\left\{u_{j_{i}}\right\}_{i \in \mathbb{N}}$ of $\left\{u_{j}\right\}_{j \in \mathbb{N}}$ such that $\left|P\left(u_{j_{i}}\right)\right|>$ 
$\varepsilon$. If $\left\{\sigma_{j}\right\}_{j \in \mathbb{N}}$ are the projections associated to $J$ and $\left\{u_{j}^{*}\right\}_{j \in \mathbb{N}}$ is the sequence of biorthogonal functionals associated to $\left\{u_{j}\right\}_{j \in \mathbb{N}}$, let $\tilde{P} \in \mathcal{P}\left({ }^{k} E\right)$ be defined by

$$
\tilde{P}(x)=\sum_{j=1}^{\infty} P\left(\sigma_{j}(x)\right)\left(u_{j}^{*}\left(\sigma_{j}(x)\right)\right)^{k-r} .
$$

It is clear that $\tilde{P}$ is a block diagonal polynomial with respect to $J$ and $\left|\tilde{P}\left(u_{j_{i}}\right)\right|=$ $\left|P\left(u_{j_{i}}\right)\right|>\varepsilon$. In the same way as in the previous proposition, we obtain the required result.

\section{Diagonal POLYNOMials}

Throughout this section $E$ is a Banach space with unconditional basis $\left\{e_{n}\right\}_{n \in \mathbb{N}}$. A polynomial $P$ is said to be diagonal if

$$
P(x+y)=P(x)+P(y)
$$

for all $x, y \in E$ with disjoint supports. The class of diagonal polynomials is a particular class of block diagonal polynomials, introduced in Section 1, for the case $J=\mathbb{N}$. We denote this class by $\mathcal{D}\left({ }^{k} E\right)$. The space of diagonal polynomials can be seen as the dual of the subspace of $\widehat{\bigotimes}_{\pi, s}^{k} E$ (the complete $k$-fold symmetric tensor product of $E$ endowed with the projective norm) spanned by the tensor diagonal basis $\left\{e_{n} \otimes \cdots \otimes e_{n}\right\}_{n \in \mathbb{N}}$. Some aspects of the tensor diagonal basis for non-symmetric tensor products were studied by Holub 21] and Arias and Farmer [1].

In the case of real Banach spaces with 1-unconditional basis considered as Banach lattices, diagonal polynomials coincide with orthogonally additive polynomials, which were widely studied for the classical Banach space $\ell_{p}$ by Sundaresan [24].

Remark 2.1. If $P$ is a diagonal polynomial on $E$, it is clear that

$$
P\left(\sum_{n=1}^{\infty} x_{n} e_{n}\right)=\sum_{n=1}^{\infty} P\left(e_{n}\right) x_{n}^{k}
$$

for all $\sum_{n=1}^{\infty} x_{n} e_{n} \in E$. Moreover, by Proposition 1.3 (consider $\sigma_{j}: E \rightarrow \overline{\left[e_{j}\right]}$, the natural projection), this series is indeed absolutely convergent, i.e. there exists $C>0$ such that

$$
\sum_{n=1}^{\infty}\left|P\left(e_{n}\right) x_{n}^{k}\right| \leq C\|x\|^{k}
$$

for all $x \in E$.

Concerning to weak sequential continuity of diagonal polynomials, for the classical space $\ell_{p}$ it is known by [24] that polynomials of a certain degree are weakly sequentially continuous whenever diagonal polynomials of the same degree are. In general, this is not the situation, as the following example shows:

Example 2.2. There exists a Banach space $E$ with unconditional basis such that $\mathcal{D}\left({ }^{k} E\right)=\mathcal{D}_{\text {wsc }}\left({ }^{k} E\right)$ for all $k \in \mathbb{N}$, but $\mathcal{P}\left({ }^{2} E\right) \neq \mathcal{P}_{\text {wsc }}\left({ }^{2} E\right)$.

Consider the example which appears in $[8]$ : $E=d_{*}(w ; 1) \times d(w ; 1)$, where $d(w ; 1)$ is a Lorentz sequence space associated to a suitable sequence $w$ and $d_{*}(w ; 1)$ is its natural predual. There, it is proved that all polynomials on both $d_{*}(w ; 1)$ and 
$d(w ; 1)$ are weakly sequentially continuous. Therefore, all diagonal polynomials on $E$ are weakly sequentially continuous. Indeed, if $P \in \mathcal{D}\left({ }^{k} E\right)$ then $P$ can be written as

$$
P\left(\sum_{n=1}^{\infty} x_{n} e_{n}, \sum_{n=1}^{\infty} y_{n} e_{n}^{*}\right)=\sum_{n=1}^{\infty} P\left(e_{n}, 0\right) x_{n}^{k}+\sum_{n=1}^{\infty} P\left(0, e_{n}^{*}\right) y_{n}^{k}
$$

for all $\left(x, x^{*}\right)=\left(\sum_{n=1}^{\infty} x_{n} e_{n}, \sum_{n=1}^{\infty} y_{n} e_{n}^{*}\right) \in E$, where $\left\{e_{n}\right\}_{n \in \mathbb{N}}\left(\right.$ resp. $\left.\left\{e_{n}^{*}\right\}_{n \in \mathbb{N}}\right)$ is the basis in $d_{*}(w ; 1)$ (resp. $d(w ; 1)$ ). Note that the above representation of the polynomial $P$ is possible since the series are absolutely convergent (see Remark 2.1). Thus $P$ is weakly sequentially continuous. Nevertheless, the polynomial $Q\left(x, x^{*}\right)=x^{*}(x)$ is not weakly sequentially continuous on $E$ (see [8] for more details).

Weak sequential continuity of diagonal polynomials has strong implications on the behavior of all polynomials against the basis, as we state in the following proposition, which is a consequence of the proof of Proposition 1.9.

Proposition 2.3. Let $E$ be a Banach space with an unconditional weakly null basis $\left\{e_{n}\right\}_{n \in \mathbb{N}}$. Assume that $\mathcal{D}\left({ }^{k} E\right)=\mathcal{D}_{w s c}\left({ }^{k} E\right)$. Then the basis $\left\{e_{n}\right\}_{n \in \mathbb{N}}$ is a $\mathcal{P}_{k}$-null sequence.

In general, the converse of Proposition 2.3 is not true, even for spaces with symmetric bases, as the following example shows:

Example 2.4. There exists a Banach space $E$ with symmetric basis $\left\{e_{n}\right\}_{n \in \mathbb{N}}$ which is $\mathcal{P}_{k}$-null for all $k \in \mathbb{N}$ but $\mathcal{D}\left({ }^{2} E\right) \neq \mathcal{D}_{\text {wsc }}\left({ }^{2} E\right)$.

Consider the Lorentz sequence space $E=d(w ; 2)$ (see e.g. [22]) associated to the sequence $w=\{1 / n\}_{n \in \mathbb{N}}$. We denote by $\left\{e_{n}\right\}_{n \in \mathbb{N}}$ the basis of $d(w ; 2)$. It is easy to check that $\sum_{i=1}^{n} 1 / i=\left\|\sum_{i=1}^{n} e_{i}\right\|^{2}$. Thus,

$$
\varliminf_{n \rightarrow \infty} \frac{\left\|\sum_{i=1}^{n} e_{i}\right\|}{n^{1 / k}}=0,
$$

which implies that $\left\{e_{n}\right\}_{n \in \mathbb{N}}$ does not admit a lower $k$-estimate for any $k \in \mathbb{N}$. By [18] the basis $\left\{e_{n}\right\}_{n \in \mathbb{N}}$ is a $\mathcal{P}_{k}$-null sequence for all $k$.

The expression

$$
P\left(\sum_{n=1}^{\infty} x_{n} e_{n}\right)=\sum_{n=1}^{\infty} \frac{1}{n} x_{n}^{2}
$$

satisfies

$$
\left|P\left(\sum_{n=1}^{\infty} x_{n} e_{n}\right)\right| \leq \sup _{\pi \in \Pi} \sum_{n=1}^{\infty} \frac{1}{n}\left|x_{\pi(n)}\right|^{2}=\left\|\sum_{n=1}^{\infty} x_{n} e_{n}\right\|^{2} .
$$

Thus, $P$ is a 2-homogeneous polynomial on $d(w ; 2)$; moreover, $P \in \mathcal{D}\left({ }^{2} d(w ; 2)\right)$. Nevertheless, $P$ is not weakly sequentially continuous. To show this, consider a normalized weakly null block sequence $\left\{u_{n}\right\}_{n \in \mathbb{N}}$ in $d(w ; 2)$ with $u_{n}=\sum_{i=p_{n}+1}^{p_{n+1}} a_{i} e_{i}$, where $\left\{p_{n}\right\}_{n \in \mathbb{N}}$ is an increasing sequence of integers, $a_{1} \geq a_{2} \geq \ldots$ and $\left(a_{n}\right)_{n \in \mathbb{N}}$ converges to zero. By [22, Prop. 4.e.3.] such a sequence has a subsequence $\left\{u_{n_{j}}\right\}_{j \in \mathbb{N}}$ equivalent to the unit vector basis of $\ell_{2}$. Thus there exists a constant $C>0$ such 
that for all $n \in \mathbb{N}$,

$$
C j \leq\left\|\sum_{i=1}^{j} u_{n_{i}}\right\|^{2}=P\left(u_{n_{1}}\right)+\cdots+P\left(u_{n_{j}}\right) .
$$

Therefore,

$$
\frac{P\left(u_{n_{1}}\right)+\cdots+P\left(u_{n_{j}}\right)}{j} \geq C,
$$

which implies that $\left\{P\left(u_{n_{j}}\right)\right\}_{j \in \mathbb{N}}$ does not converge to zero. Consequently, $P$ is not weakly sequentially continuous, as required.

We generalize Theorem 7 and Theorem 10 of 24 to the context of spaces with a basis with a lower $k$-estimate.

Proposition 2.5. Let $E$ be a Banach space with a weakly null unconditional basis $\left\{e_{n}\right\}_{n \in \mathbb{N}}$. Assume that $\left\{e_{n}\right\}_{n \in \mathbb{N}}$ has a lower $k$-estimate, for some $k \in \mathbb{N}$, and let $P \in \mathcal{D}\left({ }^{k} E\right)$. Then the following assertions are equivalent:

(i) $P$ is weakly sequentially continuous.

(ii) $\left\{P\left(e_{n}\right)\right\}_{n \in \mathbb{N}}$ converges to zero.

Proof. The part (i) implies (ii) is obvious. We show that (ii) implies (i). Assume the contrary; then $P$ is a diagonal $k$-homogeneous polynomial which is not weakly sequentially continuous. Let $\left\{u_{n}\right\}_{n \in \mathbb{N}}$ be a sequence which is weakly convergent to $u$ and such that, for some $\delta>0,\left|P\left(u_{n}\right)-P(u)\right|>\delta$ for all $n$. Consider, $u_{n}=\sum_{i=1}^{\infty} a_{i}^{(n)} e_{i}$ and $u=\sum_{i=1}^{\infty} a_{i} e_{i}$. We can assume, without loss of generality, that $\left\|u_{n}\right\| \leq 1$ for all $n$.

Since the basis has a lower $k$-estimate, there is a constant $C>0$ such that

$$
\sum_{n=1}^{\infty}\left|x_{n}\right|^{k} \leq C\left\|\sum_{n=1}^{\infty} x_{n} e_{n}\right\|^{k}
$$

The polynomial $P$ is diagonal, so $P\left(\sum_{n=1}^{\infty} x_{n} e_{n}\right)=\sum_{n=1}^{\infty} \alpha_{n} x_{n}^{k}$ with $P\left(e_{n}\right)=\alpha_{n}$.

We will show that for a given integer $N$ there is an integer $m>N$ such that $\left|\alpha_{m}\right| \geq \frac{\delta}{4 C}$, which contradicts that $\left\{P\left(e_{n}\right)\right\}_{n \in \mathbb{N}}$ converges to zero.

Let $N$ be fixed, with

$$
\begin{aligned}
\delta<\left|P\left(u_{n}\right)-P(u)\right| & \leq\left|\sum_{i=1}^{N} \alpha_{i}\left(a_{i}^{(n)}\right)^{k}-\sum_{i=1}^{N} \alpha_{i}\left(a_{i}\right)^{k}\right| \\
& +\sum_{i=N+1}^{\infty}\left|\alpha_{i}\right|\left|a_{i}^{(n)}\right|^{k}+\sum_{i=N+1}^{\infty}\left|\alpha_{i}\right|\left|a_{i}\right|^{k} \leq(\star) .
\end{aligned}
$$

Since $\lim _{n \rightarrow \infty} a_{i}^{(n)}=a_{i}$ for all $i=1, \ldots, N$, we have that, for $n$ large enough,

$$
(\star) \leq \frac{\delta}{2}+\sup _{N<i<\infty}\left|\alpha_{i}\right|\left(\sum_{i=N+1}^{\infty}\left|a_{i}^{(n)}\right|^{k}+\sum_{i=N+1}^{\infty}\left|a_{i}\right|^{k}\right) \leq \frac{\delta}{2}+2 C \sup _{N<i<\infty}\left|\alpha_{i}\right| .
$$

Thus,

$$
\frac{\delta}{2}<2 C \sup _{N<i<\infty}\left|\alpha_{i}\right|
$$


which says that there is an integer $m>N$ such that $\left|\alpha_{m}\right| \geq \frac{\delta}{4 C}$, as required. So the proof is finished.

In the light of the above result one can ask if a diagonal polynomial $P$ on a space with a weakly null basis $\left\{e_{n}\right\}_{n \in \mathbb{N}}$ is weakly sequentially continuous if and only if $\left\{P\left(e_{n}\right)\right\}_{n \in \mathbb{N}}$ converges to zero. The answer is negative in general, as Example 2.4 shows.

Proposition 2.6. Let $E$ be a Banach space with a normalized unconditional basis $\left\{e_{n}\right\}_{n \in \mathbb{N}}$ with a lower $k$-estimate with constant $C$. Then the space $\mathcal{D}\left({ }^{k} E\right)$ is $C$ isomorphic to $\ell_{\infty}$. Moreover, if the basis $\left\{e_{n}\right\}_{n \in \mathbb{N}}$ is weakly null, $\mathcal{D}_{w s c}\left({ }^{k} E\right)$ is $C$-isomorphic to $c_{0}$.

Proof. Let $T: \ell_{\infty} \rightarrow \mathcal{D}\left({ }^{k} E\right)$ be defined by

$$
T(b)(x)=\sum_{n=1}^{\infty} b_{n} x_{n}^{k} .
$$

Then, for all $b \in \ell_{\infty}$,

$$
\|b\|_{\infty} \leq\|T(b)\| \leq \sup _{\|x\| \leq 1} \sum_{n=1}^{\infty}\left|b_{n}\right|\left|x_{n}\right|^{k} \leq\|b\|_{\infty} \sup _{\|x\| \leq 1} \sum_{n=1}^{\infty}\left|x_{n}\right|^{k} \leq C\|b\|_{\infty} .
$$

And the application is surjective because if $P \in \mathcal{D}\left({ }^{k} E\right), P=T\left(\left\{P\left(e_{n}\right)\right\}_{n \in \mathbb{N}}\right)$.

Moreover, if the basis is weakly null, by Proposition 2.5 it follows that the image of $c_{0}$ by $T$ coincides with $\mathcal{D}_{w s c}\left({ }^{k} E\right)$.

For the case of spaces with a basis which dominates all of its subsequences we may obtain more precise results. Recall that a basis $\left\{e_{n}\right\}_{n \in \mathbb{N}}$ dominates all of its subsequences if there is a constant $C>0$ such that for each subsequence $\left\{e_{n_{i}}\right\}_{i \in \mathbb{N}}$ of $\left\{e_{n}\right\}_{n \in \mathbb{N}}$, and for all $m \in \mathbb{N}$,

$$
\left\|\sum_{i=1}^{m} x_{i} e_{n_{i}}\right\| \leq C\left\|\sum_{i=1}^{m} x_{i} e_{i}\right\| .
$$

Lemma 2.7. Let $E$ be a Banach space with an unconditional basis $\left\{e_{n}\right\}_{n \in \mathbb{N}}$ which dominates all of its subsequences. If $\left\{e_{n}\right\}_{n \in \mathbb{N}}$ is not a $\mathcal{P}_{k}$-null sequence, then $\left\{e_{n}\right\}_{n \in \mathbb{N}}$ has a lower $k$-estimate.

Proof. If $\left\{e_{n}\right\}_{n \in \mathbb{N}}$ is not $\mathcal{P}_{k}$-null, there are a $k$-homogeneous polynomial $P$ and a subsequence of integers $\left\{n_{j}\right\}_{j \in \mathbb{N}}$ such that $\left|P\left(e_{n_{j}}\right)\right| \geq 1$ for all $j \in \mathbb{N}$. If we define $\pi: E \rightarrow \overline{\left[\left\{e_{n_{j}}\right\}_{j \in \mathbb{N}}\right]}$ by $\pi\left(\sum_{j=1}^{\infty} x_{j} e_{j}\right)=\sum_{j=1}^{\infty} x_{j} e_{n_{j}}$, we obtain a linear continuous operator since the basis dominates its subsequences. Then, $Q=P \circ \pi$ is a $k$-homogeneous polynomial with $\left|Q\left(e_{n}\right)\right| \geq 1$ for all $n \in \mathbb{N}$. Thus,

$$
\sum_{n=1}^{\infty}\left|x_{n}\right|^{k} \leq \sum_{n=1}^{\infty}\left|Q\left(e_{n}\right)\right|\left|x_{n}\right|^{k} \leq C\left\|\sum_{n=1}^{\infty} x_{n} e_{n}\right\|^{k} .
$$

So, $\left\{e_{n}\right\}_{n \in \mathbb{N}}$ has a lower $k$-estimate, as required.

Combining Propositions 2.5] and 2.6] and Lemma 2.7], we obtain 
Corollary 2.8. Let $E$ be a Banach space with an unconditional basis $\left\{e_{n}\right\}_{n \in \mathbb{N}}$ which dominates all of its subsequences. Let

$$
N_{\infty}(E)=\inf \left\{r \in \mathbb{N}:\left\{e_{n}\right\}_{n \in \mathbb{N}} \text { is not } \mathcal{P}_{r} \text {-null }\right\} .
$$

Then, if $k \geq N_{\infty}(E)$, we have

(i) $\mathcal{D}\left({ }^{k} E\right)$ is isomorphic to $\ell_{\infty}$.

(ii) If the basis $\left\{e_{n}\right\}_{n \in \mathbb{N}}$ is weakly null, $P \in \mathcal{D}\left({ }^{k} E\right)$ is weakly sequentially continuous if and only if $\left\{P\left(e_{n}\right)\right\}_{n \in \mathbb{N}}$ converges to 0. Moreover, in this case, $\mathcal{D}_{\text {wsc }}\left({ }^{k} E\right)$ is isomorphic to $c_{0}$.

\section{An APPLication to POLYNOMial AlGEBRAS}

Throughout this section, $E$ is a real Banach space. Let $\mathcal{A}_{k}(E)$ be the algebra generated by polynomials on the unit ball of $E$ of degree at most $k$ and let $\overline{\mathcal{A}_{k}(E)}$ be its uniform closure, i.e., the smallest uniformly closed algebra containing all real valued polynomials of degree at most $k$. Obviously, $\overline{\mathcal{A}_{k}(E)} \subset \overline{\mathcal{A}_{k+1}(E)}$ for all $k \in \mathbb{N}$. The problem of whether this chain of polynomial algebras does stabilize appeared in [23], where it is solved for $\ell_{p}$ spaces. In [19] and [16], some partial answers are given for the general case. Here we clarify the situation for spaces with unconditional finite dimensional decomposition.

In the proof of the following theorem it is essential to use the result obtained in Proposition 1.8

Theorem 3.1. Let $E$ be a Banach space with unconditional finite dimensional decomposition, not containing $\ell_{1}$. Let

$$
N=\sup \left\{k \in \mathbb{N}: \mathcal{P}\left({ }^{k} E\right)=\mathcal{P}_{w s c}\left({ }^{k} E\right)\right\} .
$$

Then,

(i) If $N=\infty$,

$$
\overline{\mathcal{A}_{1}(E)}=\overline{\mathcal{A}_{2}(E)}=\ldots
$$

(ii) If $N<\infty$,

$$
\overline{\mathcal{A}_{1}(E)}=\cdots=\overline{\mathcal{A}_{N}(E)} \underset{\neq}{\subset} \overline{\mathcal{A}_{N+1}(E)} \underset{\ddagger}{\subsetneq} \overline{\mathcal{A}_{N+2}(E)} \underset{\neq}{\subsetneq} \ldots
$$

Proof. The result, in the case $N=\infty$, follows by [3] and [4]: indeed, since the space does not contain $\ell_{1}$, all polynomials are weakly uniformly continuous on bounded sets and $\overline{\mathcal{A}_{k}(E)}=\overline{\mathcal{A}_{1}(E)}$ for all $k \in \mathbb{N}$.

In the case $N<\infty$, by the same argument as above we have

$$
\overline{\mathcal{A}_{1}(E)}=\cdots=\overline{\mathcal{A}_{N}(E)} .
$$

Assume that for some $k \geq N$ we have $\overline{\mathcal{A}_{k}(E)}=\overline{\mathcal{A}_{k+1}(E)}$. Since $k+1>N$, there is a polynomial of degree at most $k+1$ which is not weakly sequentially continuous. By Corollary 1.7, we may assume that there is a $(k+1)$-homogeneous polynomial which is not weakly sequentially continuous at 0 . Then, by using Proposition 1.8 there are a seminormalized weakly null block sequence $\left\{u_{n}\right\}_{n \in \mathbb{N}}$ and a polynomial $P$ such that

$$
P\left(\sum_{n=1}^{\infty} a_{n} u_{n}\right)=\sum_{n=1}^{\infty} a_{n}^{k+1}
$$

for all $\sum_{n=1}^{\infty} a_{n} u_{n} \in \overline{\left[\left\{u_{n}\right\}_{n \in \mathbb{N}}\right]}$. 
We proceed as in [19], adopting the spreading model ideas developed in [16]. Thus, there are $n(k) \in \mathbb{N}$ and $\epsilon>0$ such that

$$
\sup \left\{\left|f\left(x_{1}, \ldots, x_{n(k)+1}\right)-\sum_{j=1}^{n(k)+1} x_{j}^{k+1}\right|: \sum_{j=1}^{n(k)+1}\left|x_{j}\right| \leq 1\right\} \geq \epsilon,
$$

for every $f \in \mathcal{S}_{k}\left(\mathbb{R}^{n(k)+1}\right)$. By $\mathcal{S}_{k}\left(\mathbb{R}^{n(k)+1}\right)$ we mean the algebra generated by the subsymmetric polynomials on $\mathbb{R}^{n(k)+1}$ of degree at most $k$ (see 19 for more details). Since $P \in \overline{\mathcal{A}_{k}(E)}$, there exists $Q \in \mathcal{A}_{k}(E)$ such that

$$
\sup _{\|x\| \leq C}|P(x)-Q(x)|<\frac{\epsilon}{4}
$$

where $\epsilon$ is defined above and $C>0$ is such that $\left\|u_{n}\right\| \leq C$ for all $n$. Using spreading model techniques as in [16], we obtain a finite sequence $\left\{u_{i_{1}}, \ldots, u_{i_{n(k)+1}}\right\}$ such that $\left.Q\right|_{\left[u_{i_{1}}, \ldots, u_{i_{n}(k)+1}\right]}$ can be approximated by $\tilde{Q} \in \mathcal{S}_{k}\left(\mathbb{R}^{n(k)+1}\right)$ within $\frac{\epsilon}{4}$ in the ball $B(0 ; C)$. Thus,

$$
\sup \left\{\left|\sum_{j=1}^{n(k)+1} x_{j}^{k+1}-\tilde{Q}\left(x_{1}, \ldots, x_{n(k)+1}\right)\right|:\left\|\mid \sum_{j=1}^{n(k)+1} x_{j} u_{i_{j}}\right\| \leq C\right\} \leq \frac{\epsilon}{2},
$$

which contradicts (4).

For spaces containing $\ell_{1}$ the following result was proved in [19, Cor. 7]:

Proposition 3.2. Let $E$ be a Banach space containing $\ell_{1}$. Then,

$$
\overline{\mathcal{A}_{1}(E)} \underset{\neq}{\subset} \overline{\mathcal{A}_{2}(E)} \underset{\neq}{\subset} \ldots
$$

Remark 3.3. Note that associated to each linear continuous operator $T: E \rightarrow \ell_{k}$, where $k<\infty$, there is a $k$-homogeneous polynomial $P$ on $E$ defined by

$$
P(x)=\sum_{n=1}^{\infty} x_{n}^{*}(x)^{k}
$$

where $x_{n}^{*}(x)=T(x)_{n}$ (the $n$-th coordinate of $\left.T(x)\right)$.

Moreover, for spaces not containing $\ell_{1}$, the existence of a non-compact operator $T: E \rightarrow \ell_{k}$ is equivalent to the existence of a linear operator $\tilde{T}: E \rightarrow \ell_{k}$ which takes a weakly null sequence into the unit vector basis of $\ell_{k}$ (see e.g. [19]). Both conditions imply the existence of a non-weakly-sequentially continuous $k$-homogeneous polynomial (see Proposition 3.4 below). We do not know whether the converse is true.

Problem 1. Let $E$ be a Banach space not containing $\ell_{1}$ which admits a $k$-homogeneous polynomial which is not weakly sequentially continuous. Then does there exist a linear non-compact operator from $E$ into $\ell_{k}$ ?

Concerning this problem, it is obvious that the existence of a non-weaklysequentially continuous polynomial is equivalent to the existence of a non- $\mathcal{P}_{k}$-null sequence, for a certain $k$. We now relate non- $\mathcal{P}_{k}$-null sequences to non-compact operators from $E$ into $\ell_{k}$ : 
Proposition 3.4. Let $\left\{u_{n}\right\}_{n \in \mathbb{N}}$ be a weakly null normalized sequence in a Banach space $E$ and $k \in \mathbb{N}$. If there is a linear continuous mapping $T: E \rightarrow \ell_{k}$ such that

$$
\varlimsup_{n \rightarrow \infty}\left\|T\left(u_{n}\right)\right\|>0
$$

then $\left\{u_{n}\right\}_{n \in \mathbb{N}}$ is not $\mathcal{P}_{k}$-null.

Moreover, if $\left\{u_{n}\right\}_{n \in \mathbb{N}}$ is an unconditional basis of $E$, the converse is also true.

Proof. First, since the sequence $\left\{T\left(u_{n}\right)\right\}_{n \in \mathbb{N}} \subset \ell_{k}$ is weakly null but not norm null, we may extract a subsequence $\left\{T\left(u_{n_{j}}\right)\right\}_{j \in \mathbb{N}}$ which is equivalent to the vector unit basis of $\ell_{k}$ (let $\Lambda: \overline{\left[\left\{T\left(u_{n_{j}}\right)\right\}_{j \in \mathbb{N}}\right]} \rightarrow \ell_{k}$ be the isomorphism) and spans a complemented subspace in $\ell_{k}$ (via a projection $\pi: \ell_{k} \rightarrow \overline{\left[\left\{T\left(u_{n_{j}}\right)\right\}_{j \in \mathbb{N}}\right]}$ ). Consider the $k$-homogeneous polynomial $P$ associated to $\Lambda \circ \pi \circ T$, as in Remark 3.3 , that is,

$$
P(x)=\sum_{i=1}^{\infty}((\Lambda \circ \pi \circ T)(x))_{i}^{k} .
$$

Thus $P\left(u_{n_{j}}\right)=1$ for all $j$; hence $\left\{u_{n}\right\}_{n \in \mathbb{N}}$ is not $\mathcal{P}_{k}$-null.

On the other hand, if $\left\{u_{n}\right\}_{n \in \mathbb{N}}=\left\{e_{n}\right\}_{n \in \mathbb{N}}$ is an unconditional basis in $E$ which is not $\mathcal{P}_{k}$-null, by Proposition 2.3 there is a $k$-homogeneous diagonal polynomial with $\left|P\left(e_{n_{j}}\right)\right| \geq 1$, where $\left\{n_{j}\right\}_{j \in \mathbb{N}}$ is a subsequence of integers, and

$$
\sum_{n=1}^{\infty}\left|P\left(e_{n}\right) x_{n}^{k}\right| \leq C\|x\|^{k}
$$

for all $x \in E$. Thus,

$$
\sum_{j=1}^{\infty}\left|x_{n_{j}}\right|^{k} \leq C\|x\|^{k}
$$

for all $x \in E$. So, $T: E \rightarrow \ell_{k}$ defined by $T(x)=\left(x_{n_{j}}\right)_{j \in \mathbb{N}}$ is a linear continuous operator which takes $\left\{e_{n_{j}}\right\}_{j \in \mathbb{N}}$ into the unit vector basis of $\ell_{k}$.

The converse of Proposition 3.4 is not true in general. To show this we use the following remark.

Remark 3.5. Let $E$ and $F$ be Banach spaces and $T: E \times F \rightarrow \ell_{2}$ a linear continuous mapping. Associated to it, we define linear continuous mappings $T_{1}: E \rightarrow \ell_{2}$ and $T_{2}: F \rightarrow \ell_{2}$ by

$$
T_{1}(u)=T(u, 0) \quad \text { and } \quad T_{2}(v)=T(0, v)
$$

for all $u \in E, v \in F$; thus, $T(u, v)=T_{1}(u)+T_{2}(v)$. This implies that for a given sequence $\left\{\left(u_{n}, v_{n}\right)\right\}_{n \in \mathbb{N}}$ in $E \times F$ such that $\varlimsup_{n \rightarrow \infty}\left\|T\left(u_{n}, v_{n}\right)\right\|>0$ we have either $\varlimsup_{n \rightarrow \infty}\left\|T_{1}\left(u_{n}\right)\right\|>0$ or $\varlimsup_{n \rightarrow \infty}\left\|T_{2}\left(v_{n}\right)\right\|>0$.

Example 3.6. There is a non- $\mathcal{P}_{2}$-null normalized sequence $\left\{u_{n}\right\}_{n \in \mathbb{N}}$ in a Banach space $E$ with unconditional basis, such that for all linear continuous mappings $T$ : $E \rightarrow \ell_{2}$ we have $\lim _{n \rightarrow \infty}\left\|T\left(u_{n}\right)\right\|=0$.

Let $\ell_{M}$ be the Orlicz sequence space associated to an Orlicz function $M$ satisfying

$$
\varliminf_{t \rightarrow 0} \frac{M(t)}{t^{2}}=0, \quad \varlimsup_{t \rightarrow 0} \frac{M(t)}{t^{2}}=\infty .
$$

It is possible to construct such an Orlicz function (see e.g. [20] for details) even with Boyd indexes $\alpha_{M}=\beta_{M}=2$. Denote as usual by $\left\{e_{n}\right\}_{n \in \mathbb{N}}\left(\right.$ resp. $\left\{e_{n}^{*}\right\}_{n \in \mathbb{N}}$ ) 
the basis of $\ell_{M}$ (resp. $\ell_{M}^{*}$ ). By [17] it follows that $\left\{e_{n}\right\}_{n \in \mathbb{N}}$ has neither lower nor upper 2-estimates, which implies that both $\left\{e_{n}\right\}_{n \in \mathbb{N}}$ and $\left\{e_{n}^{*}\right\}_{n \in \mathbb{N}}$ do not admit a lower 2-estimate. So both bases are $\mathcal{P}_{2}$-null and the sequence $\left\{\left(e_{n}, e_{n}^{*}\right)\right\}_{n \in \mathbb{N}}$ is not $\mathcal{P}_{2}$-null in $\ell_{M} \times \ell_{M}^{*}$ (consider again $\left.P\left(x, x^{*}\right)=x^{*}(x)\right)$. Nevertheless, there is no $T: \ell_{M} \times \ell_{M}^{*} \rightarrow \ell_{2}$ such that

$$
\varlimsup_{n \rightarrow \infty}\left\|T\left(e_{n}, e_{n}^{*}\right)\right\|>0 .
$$

Otherwise, by Remark 3.5 and Proposition 3.4 we would conclude that either $\left\{e_{n}\right\}_{n \in \mathbb{N}}$ or $\left\{e_{n}^{*}\right\}_{n \in \mathbb{N}}$ is not $\mathcal{P}_{2}$-null, which is not possible.

We finish our discussions of Problem 1 by connecting it to another open problem posed in [10.

Problem 2. Does there exist a reflexive infinite dimensional Banach space $E$ such that both $\mathcal{P}\left({ }^{k} E\right)=\mathcal{P}_{w s c}\left({ }^{k} E\right)$ and $\mathcal{P}\left({ }^{k} E^{*}\right)=\mathcal{P}_{w s c}\left({ }^{k} E^{*}\right)$, for a certain integer $k>1$ ?

Note that for non-reflexive spaces a positive answer to Problem 2 was given in 8. The non-symmetric version of this question has a great interest in the case $k=2$. Indeed, it is equivalent to asking for the existence of an infinite dimensional Banach space such that $\mathcal{L}\left(E, E^{*}\right)=\mathcal{K}\left(E, E^{*}\right)$ and $\mathcal{L}\left(E^{*}, E\right)=\mathcal{K}\left(E^{*}, E\right)$ (as usual, $\mathcal{K}\left(E, E^{*}\right)$ denotes the space of compact operators from $E$ into $\left.E^{*}\right)$.

Remark 3.7. We would like to point out that an affirmative answer of Problem 1 would give a negative answer to Problem 2. Indeed, assume $E$ is a reflexive Banach space such that $\mathcal{P}\left({ }^{2} E\right)=\mathcal{P}_{w s c}\left({ }^{2} E\right)$ and $\mathcal{P}\left({ }^{2} E^{*}\right)=\mathcal{P}_{w s c}\left({ }^{2} E^{*}\right)$. Then the polynomial $P: E \times E^{*} \rightarrow \mathbb{R}$ defined by $P\left(x, x^{*}\right)=x^{*}(x)$ is not weakly sequentially continuous; nevertheless, every linear continuous mapping $T: E \times E^{*} \rightarrow \ell_{2}$ is compact. Otherwise, it would follow by Remark 3.5 that there is a non-compact operator on either $E$ or $E^{*}$, which is not possible. On the other hand, a negative answer to Problem 1 would give an idea of where to find an example for Problem 2 .

\section{ACKNOWLEDGMENTS}

We would like to thank Professor F. Hernández for his help with Orlicz spaces, and Professor J. A. Jaramillo for valuable and helpful conversations concerning this work. The first author wishes to thank the Departamento de Análisis Matemático of Universidad Complutense de Madrid for its hospitality during the period December 1997-February 1998, when this work was initiated.

\section{REFERENCES}

[1] A. Arias and J. Farmer, On the structure of tensor products of $\ell_{p}$-spaces, Pacific. J. Math. 175, 1 (1996), 13-37. MR 98d:46015

[2] R. Aron and J. Globevnik, Analytic functions on $c_{o}$, Rev. Mat. Univ. Complut. Madrid 2 (1989), suppl., 27-33. MR 91d:46051

[3] R. Aron, C. Hervés and M. Valdivia, Weakly continuous mappings on Banach spaces, J. Funct. Anal. 52 (1983), 189-204. MR 84g:46066

[4] R. Aron and J. Prolla, Polynomial approximation of differentiable functions on Banach spaces, J. Reine Angew. Math. 3 (1980), 195-216. MR 81c:41078

[5] J. Bochnak and J. Siciak, Polynomials and multilinear mappings in topological vector spaces, Studia Math. 39 (1971), 59-76. MR 47:2364

[6] F. Bombal and M. Fernández-Unzueta, Polynomial convergence of sequences in Banach spaces, Rev. Acad. Cienc. Exact. Fís. Natur. Madrid, to appear. 
[7] C. Boyd and R. Ryan, Bounded weak continuity of homogeneous polynomials at the origin, Arch. Math. (Basel) 71, 3 (1998), 211-218. MR 99k:46076

[8] J. Castillo, R. García and R. Gonzalo, Banach spaces in which all multilinear forms are weakly sequentially continuous, Studia Math. 136, 2 (1999), 121-145. CMP 2000:02

[9] V. Dimant and S. Dineen, Banach subspaces of spaces of holomorphic functions and related topics, Math. Scand. 83 (1998), 142-160. MR 99m:46031

[10] V. Dimant and I. Zalduendo, Bases in spaces of multilinear forms over Banach spaces, $J$. Math. Anal. Appl. 200 (1996), 548-566. MR 97k:96017

[11] S. Dineen, "Complex analysis in locally convex spaces", North Holland Math. Studies 57, 1981. MR 84b:46050

[12] S. Dineen, A Dvoretzky theorem for polynomials, Proc. Amer. Math. Soc. 123, (1995), 28172821. MR 95k:46021

[13] S. Dineen, "Complex analysis on Infinite Dimensional Spaces", Springer Monographs in Mathematics, 1999. CMP 99:17

[14] S. Dineen and M. Lindström, Spaces of homogeneous polynomials containing $c_{0}$ or $\ell_{\infty}$, on "Functional Analysis", de Gruyter, Berlin, 1996, p. 119-127. MR 97i:46083

[15] J. Farmer and W. Johnson, Polynomial Schur and polynomial Dunford-Pettis properties, Contemp. Math. 144 (1993), 95-105. MR 94e:46078.

[16] R. Gonzalo, Multilinear forms, subsymmetric polynomials, and spreading models on Banach spaces, J. Math. Anal. Appl. 202 (1996), 379-397. MR 98a:46054

[17] R. Gonzalo, Upper and lower estimates in Banach sequence spaces, Comment. Math. Univ. Carolin. 36, 4 (1995), 641-653. MR 97d:46020

[18] R. Gonzalo, Smoothness and polynomials on Banach spaces, Doctoral Thesis, Universidad Complutense de Madrid, 1994.

[19] P. Hájek, Polynomial algebras on classical Banach spaces, Israel J. Math. 106 (1998), 209-220. MR 99k:46079

[20] F. Hernández and V. Peirats, Weighted sequence subspaces of Orlicz function spaces isomorphic to $\ell_{p}$, Arch. Math. (Basel) (1987), 270-280. MR 89c:46044

[21] J. R. Holub, Tensor product bases and tensor diagonals, Trans. Amer. Math. Soc. 151 (1970), 563-579. MR 43:5286

[22] J. Lindenstrauss and L. Tzafriri, "Classical Banach spaces I", Springer, 1977. MR 58:17766

[23] A. Nemirovskii and S. Semenov, The polynomial approximation of functions on Hilbert spaces, Math. USSR-Sb 21 (1973), 255-273. MR 58:30211b

[24] K. Sundaresan, Geometry of spaces of homogeneous polynomials on Banach lattices, Applied geometry and discrete mathematics, 571-586, DIMACS Ser. Discrete Math. Theoret. Comput. Sci., 4, Amer. Math. Soc., Providence, RI, 1991. MR 92k:46077

Departamento de Economía y Matemática, Universidad de San Andrés, Vito Dumas 284 (1644) Victoria, Prov. de Buenos Aires, Argentina

E-mail address: vero@udesa.edu.ar

Departamento de Análisis Matemático, Facultad de Ciencias Matemáticas, Universidad Complutense, 28040 Madrid, Spain

Current address: Departamento de Matemática Aplicada, Facultad de Informática, Universidad Politécnica, Campus de Montegancedo, 28660 Boadilla del Monte, Madrid, Spain

E-mail address: rngonzalo@fi.upm.es 